\title{
Gain-loss based convex risk limits in discrete-time trading
}

\author{
Mustafa Ç. Pınar
}

Received: 19 June 2009 / Accepted: 3 June 2010 / Published online: 15 June 2010

(C) Springer-Verlag 2010

\begin{abstract}
We present an approach for pricing and hedging in incomplete markets, which encompasses other recently introduced approaches for the same purpose. In a discrete time, finite space probability framework conducive to numerical computation we introduce a gain-loss ratio based restriction controlled by a loss aversion parameter, and characterize portfolio values which can be traded in discrete time to acceptability. The new risk measure specializes to a well-known risk measure (the Carr-GemanMadan risk measure) for a specific choice of the risk aversion parameter, and to a robust version of the gain-loss measure (the Bernardo-Ledoit proposal) for a specific choice of thresholds. The result implies potentially tighter price bounds for contingent claims than the no-arbitrage price bounds. We illustrate the price bounds through numerical examples from option pricing.
\end{abstract}

Keywords Incomplete markets · Acceptability · Martingale measure · Contingent claim · Pricing

\section{Introduction}

The purpose of the present paper is to address an important problem in financial economics: the pricing of financial instruments called "contingent claims" in incomplete

\footnotetext{
The research for the paper was carried out while I was on leave at the Department of Operations Research and Financial Engineering Princeton University, Princeton, NJ 08544. I am indebted to Savaş Dayanık for valuable discussions, and for suggesting the current form of the main result. This research is partially supported by TUBITAK grant 107K250. I am also grateful for the support of the USA Fulbright Commission.
}

M. Ç. Pınar ( $\varangle)$

Department of Industrial Engineering, Bilkent University, 06800 Ankara, Turkey

e-mail: mustafap@bilkent.edu.tr 
markets. In an incomplete financial market which does not allow arbitrage opportunities it is well-known that there exists a set of "risk neutral" probability measures that make the (discounted) prices of traded instruments martingales. The set of risk neutral measures has the property that the value of the cheapest portfolio to dominate the pay-off at maturity of a contingent claim coincides with the maximum expected value of the (discounted) pay-off of the claim with respect to this set. This value, called the "super-hedging price", allows the seller to assemble a portfolio that achieves a value at least as large as the pay-off to the claim holder at the maturity date of the claim in all non-negligible events. The super-hedging price is the natural price to be asked by the writer of a contingent claim and, together with the bid price obtained by considering the analogous problem from the point of view of the buyer, constitutes an interval which is sometimes called the "no-arbitrage price interval" for the claim in question. However, these prices may not be useful in practice; see chapters 7 and 8 of Föllmer and Schied (2004) for a discussion and examples showing that the super-hedging price may be too high both from a theoretical and practical point of view.

Bernardo and Ledoit (2000) introduced the gain-loss ratio for pricing and hedging contingent claims in incomplete financial markets. This approach advocates ruling out investment opportunities with a certain expected gain-loss ratio instead of ruling out only the arbitrage opportunities from financial markets since an investment opportunity with a reasonably high expected gain to loss ratio can be acceptable to many investors although it is not an arbitrage opportunity. This requirement of eliminating not only arbitrage but also gain-loss ratio opportunities from the market translates as a restriction in the dual space of martingale (pricing) measures, and leads to potentially tighter pricing bounds on contingent claims in incomplete markets. The Bernardo and Ledoit gain-loss pricing criterion was developed in a static, one-step (two period, single trading point) framework and it is easy to see that it depends on the objective probability measure that governs the market (in contrast to no-arbitrage pricing theory which does not depend on this measure except for sets of measure zero). The Bernardo and Ledoit gain-loss ratio criterion is an example of acceptable investment opportunities as discussed in Carr et al. (2001). According to Carr et al., while arbitrage opportunities are absolutely acceptable to every investor, the continuity of preferences leads to existence and acceptability (by a wide variety of investors) of mildly risky investment opportunities. The acceptability criterion introduced by Carr et al. (2001), and to which we also refer to as the Carr-Madan-Geman risk constraint, is as follows. Whether an investor holds a particular portfolio is determined by specifying a set of probability measures and associated floors which expected portfolio values must exceed to be deemed acceptable. Under the assumption that the liquid assets are priced so that each portfolio of assets has negative expected return under at least one measure, Carr et al. derived a counterpart to the first fundamental theorem of asset pricing. While Carr et al. worked in a single period finite probability setting (although they indicate an infinite state extension), Larsen et al. (2004) characterized the set of random variables that can be traded continuously to acceptability at a fixed future date according to the acceptability criterion of Carr et al.

Pricing in incomplete markets using convex and coherent risk measures is a subject that is related to the present paper and continues to receive attention in the literature. To cite a few examples, Cherny (2007) provides explicit expressions for superhedging and 
subhedging prices of European options in discrete time markets using coherent risk measures. In Cherny (2007) the fundamental theorem of asset pricing is proved using the concept of a "good-deal" (a good-deal is defined by Cochrane and Saa Requejo (2000) as an investment opportunity with a high Sharpe ratio) under coherent risk measures. Extension to transaction costs is also given. Cerny and Hodges (2000) and Cerny (2003) present a theory of good-deal pricing, and develops a Sharpe-ratio based measure for pricing contingent claims in incomplete markets while Staum (2004) provides a theory of good-deal pricing in general spaces. Jaschke and Küchler (2001) study the link between good-deal pricing and risk measures. Björk and Slinko (2006) extend the theory of good-deal pricing to jump processes. Roorda et al. (2005) extend the theory of coherent risk measures to a dynamic setting while Klöppel and Schweizer (2007) develop a pricing approach based on indifference pricing using conditionally convex risk measures in dynamic, incomplete markets. Kallsen (2000) and Oberman and Zariphopoulou (2003) also treat utility based pricing in incomplete markets. Xu (2006) also develops a theory of pricing in incomplete markets using convex risk measures.

In the present paper, we develop a risk measure closely related to the aforementioned ideas in a discrete time, multi-period, finite probability setting while generalizing the gain-loss ratio constraint, inspired by a more recent contribution of Larsen et al. (2004). In particular, we give a version of the gain-loss ratio criterion akin to the pricing framework of Carr et al. (2001) and Larsen et al. (2004), which reduces to their results under a specific choice of a loss aversion parameter. On the other hand, for a trivial choice of "risk floors", we obtain a version of the gain-loss ratio risk measure of Bernardo and Ledoit under several trial measures, i.e., a robust version of the gain-loss ratio which alleviate to some extent the dependence on the original measure. Furthermore, the main result of the paper implies potentially tighter price bounds for contingent claims than the no-arbitrage price bounds. These bounds can be computed by solving linear programming problems which are routinely processed by modern algorithms and software. The price bounds may be considerably sharper than the no-arbitrage price bounds as illustrated with numerical examples. The risk measure given in the present paper is not, strictly speaking, a coherent risk measure since it violates the translation invariance property of coherent risk measures (Ruszczynski and Shapiro 2006).

The present paper is also related to two previous papers of the author (Pinar et al. 2010; Pinar 2010). In Pinar et al. (2010) a gain-loss ratio based pricing as in Bernardo and Ledoit is developed in incomplete, discrete time, finite state markets, and extended to cover proportional transaction costs. In Pinar (2010), the gain-loss ratio is treated in a static (single period) but infinite state probability context where the probability measure can vary in a suitable uncertainty set defined by upper and lower limiting measures, or alternatively by a Bregman like distance restriction with respect to a central measure. The gain-loss risk criterion of the present paper generalizes the gain-loss restriction treated in Pinar et al. (2010) and Pinar (2010).

The unifying theme in all the aforementioned approaches is to obtain a restriction on the set of admissible risk-neutral measures, and therefore yield sharper price bounds compared to no-arbitrage pricing while incorporating investor preferences, thus bridging the gap between arbitrage pricing theory and expected utility theory. It turns out 
that the notion of gain-loss measure is also related to prospect theory of Kahneman and Tversky (1979) proposed as an alternative to expected utility framework. In prospect theory, it is presumed based on experimental evidence that gains and losses have asymmetric effects on the agents' welfare where welfare, or utility, is defined not over total wealth but over gains and losses; see Grüne and Semmler (2008) and Barberis et al. (2001) for details on the use of the gain-loss function as a central part of welfare functions in asset pricing.

This paper is organized as follows. After some preliminaries in Sect. 2 we state and prove the main result of the paper in Sect. 3. The result is specialized to the risk measures of Carr et al. (2001) and Larsen et al. (2004), and that of Bernardo and Ledoit (2000), respectively in Sects. 4 and 5. The implications of the main result for time $t=0$, and pricing of contingent claims are investigated in Sects. 6 and 7, respectively. We illustrate the results of the paper with examples from pricing a European call option in Sect. 8.

\section{Preliminaries}

We assume as in King (2002) that security prices and other payments are discrete random variables supported on a finite probability space $(\Omega, \mathcal{F}, \mathbb{P})$ whose atoms are sequences of real-valued vectors (asset values) over discrete time periods $t=0,1, \ldots, T$. We further assume the market evolves as a discrete, non-recombinant scenario tree (hence, suitable for incomplete markets) in which the partition of probability atoms $\omega \in \Omega$ generated by matching path histories up to time $t$ corresponds one-to-one with nodes $n \in \mathcal{N}_{t}$ at level $t$ in the tree. The set $\mathcal{N}_{0}$ consists of the root node $n=0$, and the leaf nodes $n \in \mathcal{N}_{T}$ correspond one-to-one with the probability atoms $\omega \in \Omega$. While not needed in the finite probability setting, the $\sigma$-algebras $\mathcal{F}_{t}$ generated by the partitions $\mathcal{N}_{t}$ are such that, $\mathcal{F}_{0}=\{\emptyset, \Omega\}, \mathcal{F}_{t} \subset \mathcal{F}_{t+1}$ for all $0 \leq t \leq T-1$ and $\mathcal{F}_{T}=\mathcal{F}$. A stochastic process is said to be $\left(\mathcal{F}_{t}\right)_{t=0}^{T}$-adapted if for each $t=0, \ldots, T$, the outcome of the process only depends on the element of $\mathcal{F}_{t}$ that has been realized at stage $t$. Similarly, a decision process is said to be $\left(\mathcal{F}_{t}\right)_{t=0}^{T}$-adapted if for each $t=0, \ldots, T$, the decision depends on the element of $\mathcal{F}_{t}$ that has been realized at stage $t$. In the scenario tree, every node $n \in \mathcal{N}_{t}$ for $t=1, \ldots, T$ has a unique parent denoted $a(n) \in \mathcal{N}_{t-1}$, and every node $n \in \mathcal{N}_{t}, t=0,1, \ldots, T-1$ has a non-empty set of child nodes $\mathcal{C}(n) \subset \mathcal{N}_{t+1}$. We denote the set of all nodes in the tree by $\mathcal{N}$, and $t(n)$ encodes the time period to which node $n$ belongs. The set $\mathcal{A}(n, m)$ denotes the collection of ascendant nodes or the unique path leading to node $n$ (including itself) from node $m$. The probability distribution $\mathbb{P}$ is obtained by attaching positive weights $p_{n}$ to each leaf node $n \in \mathcal{N}_{T}$ so that $\sum_{n \in \mathcal{N}_{T}} p_{n}=1$. For each non-leaf (intermediate level) node in the tree we have, recursively,

$$
p_{n}=\sum_{m \in \mathcal{C}(n)} p_{m}, \quad \forall n \in \mathcal{N}_{t}, t=T-1, \ldots, 0 .
$$

Hence, each non-leaf node has a probability mass equal to the combined mass of its child nodes. 
A random variable $X$ is a real valued function defined on $\Omega$. It can be lifted to the nodes of a partition $\mathcal{N}_{t}$ of $\Omega$ if each level set $\left\{X^{-1}(a): a \in \mathbb{R}\right\}$ is either the empty set or is a finite union of elements of the partition. In other words, $X$ can be lifted to $\mathcal{N}_{t}$ if it can be assigned a value on each node of $\mathcal{N}_{t}$ that is consistent with its definition on $\Omega$. This kind of random variable is said to be measurable with respect to the information contained in the nodes of $\mathcal{N}_{t}$. A stochastic process $\left\{X_{t}\right\}$ is a time-indexed collection of random variables such that each $X_{t}$ is measurable with respect $\mathcal{N}_{t}$. We use the notation $X_{t}^{n}$ to denote the value of the stochastic process $X_{t}$ at time $t$ and node $n \in \mathcal{N}_{t}$. The expected value of $X_{t}$ is defined by the sum

$$
\mathbb{E}^{\mathbb{P}}\left[X_{t}\right]:=\sum_{n \in \mathcal{N}_{t}} p_{n} X_{t}^{n}
$$

The conditional expectation of $X_{t+1}$ on $\mathcal{N}_{t}$ is given by the expression

$$
\mathbb{E}^{\mathbb{P}}\left[X_{t+1} \mid \mathcal{N}_{t}\right]:=\sum_{m \in \mathcal{C}(n)} \frac{p_{m}}{p_{n}} X_{t}^{m} .
$$

The market consists of a single traded security with prices at time $t$ and node $n \in \mathcal{N}_{t}$ given by the scalar $S_{t}^{n}$. The number of shares of security held by the investor in state (node) $n \in \mathcal{N}_{t}$ at time $t$ is denoted $\pi_{t}^{n}$. Therefore, $\left\{\pi_{t}\right\}$ is an adapted portfolio process whose value at each state $n \in \mathcal{N}_{t}$ is $\pi_{t}^{n} \in \mathbb{R}$. The value of the portfolio at state $n$ is $S_{t}^{n} \pi_{t}^{n}$. The assumption of a single security can be easily relaxed, and the development of the paper can be repeated for multiple securities, mutatis mutandis.

Definition 1 If there exists a probability measure $\mathbb{Q}=\left\{q_{n}\right\}_{n \in \mathcal{N}_{T}}$ such that

$$
S_{t}=\mathbb{E}^{\mathbb{Q}}\left[S_{t+1} \mid \mathcal{N}_{t}\right](t \leq T-1)
$$

then the process $\left\{S_{t}\right\}$ is called a martingale under $\mathbb{Q}$, and $\mathbb{Q}$ is called a martingale probability measure for the process.

We denote by $\mathrm{Q}$ the set of all probability measures that make $S$ a martingale over $[0,1, \ldots, T]$, and use $\cos$ to denote the convex hull of a set $S$.

We have a finite number of scenario measures (also referred to as trial measures) $\mathbb{P}^{i}, i=1, \ldots, d$ which may or may not include $\mathbb{P}$. Furthermore, we have $d$ floors $f^{i} \in \mathbb{R}$ associated with each measure $\mathbb{P}^{i}$. Note that some (or all) of the $f^{i}$ s can be negative. In particular, Carr-Madan-Geman distinguished between valuation measures associated with a floor equal to zero, and stress measures which attach increased weight to states-of-nature corresponding to potential losses, with associated negative floors with a view to limit losses in those states of the world. We denote by $f^{\mathbb{Q}}$ the quantity $\sup \left\{\sum^{c} \alpha^{i} f^{i} \mid \mathbb{Q}=\sum^{c} \alpha^{i} \mathbb{P}^{i}\right\}$, where $\sum^{c}$ denotes convex combination.

\section{The main result}

The point of departure of Carr et al. (2001) is that an arbitrage opportunity is something acceptable to every investor. However, it must also be true that opportunities 
carrying a mild risk exist in the market, and are acceptable to some investors. We define the following notion of acceptability, referred to as $\lambda$-acceptability. A portfolio value is deemed $\lambda$-acceptable $(\lambda \geq 1)$ if the expected positive portfolio value reached at a future date (after trading) exceeds the sum of some predetermined lower bound (depending on the chosen measure) and $\lambda$ times the expected negative portfolio value, under all given probability measures. I.e., if $Z$ represents a financial position at time $T$ in the future we define $\lambda$-acceptability as satisfaction of the system of inequalities of the type $\mathbb{E}^{\mathbb{P}^{i}}\left[Z_{+}-\lambda Z_{-}\right] \geq f^{i}$.

More precisely, choose $\lambda \geq 1$, and fix $\bar{t} \in\{0,1, \ldots, T-1\}$. We declare random variable $X$, measurable with respect to $\mathcal{N}_{\bar{t}}, \lambda$-acceptable at time $\bar{t}$ if there exists a trading strategy $\Pi=\left\{\pi_{t}\right\}, t \in\{\bar{t}, \bar{t}+1, \ldots, T-1\}$ such that

$$
\begin{aligned}
\mathbb{E}^{\mathbb{P}^{i}} & {\left[\left(X+\sum_{t=\bar{t}}^{T-1} \pi_{t}\left(S_{t+1}-S_{t}\right)\right)_{+}\right] } \\
& -\lambda \mathbb{E}^{\mathbb{P}^{i}}\left[\left(X+\sum_{t=\bar{t}}^{T-1} \pi_{t}\left(S_{t+1}-S_{t}\right)\right)_{-}\right] \geq f^{i} \quad \forall i=1, \ldots, d .
\end{aligned}
$$

Let $\mathcal{G}_{\bar{t}}(\lambda)$ be the set of all random variables $X$ measurable with respect to $\mathcal{N}_{\bar{t}}$ that are $\lambda$-acceptable at time $\bar{t}$. It is easy to verify that $\mathcal{G}_{\bar{t}}(\lambda)$ is a convex set.

Proposition 1 The set $\mathcal{G}_{\bar{t}}(\lambda)$ is convex.

Proof Let $X \in \mathcal{G}_{\bar{t}}(\lambda)$. Then there exists a trading strategy $\Pi=\left\{\pi_{t}\right\}, t \in\{\bar{t}, \bar{t}+$ $1, \ldots, T-1\}$ such that

$$
\begin{aligned}
\mathbb{E}^{\mathbb{P}^{i}} & {\left[\left(X+\sum_{t=\bar{t}}^{T-1} \pi_{t}\left(S_{t+1}-S_{t}\right)\right)_{+}\right] } \\
& -\lambda \mathbb{E}^{\mathbb{P}^{i}}\left[\left(X+\sum_{t=\bar{t}}^{T-1} \pi_{t}\left(S_{t+1}-S_{t}\right)\right)_{-}\right] \geq f^{i} \quad \forall i=1, \ldots, d .
\end{aligned}
$$

Since the function $\mathbb{E}\left[Z_{+}-\lambda Z_{-}\right]$is concave in $Z$ for $\lambda \geq 1$, and

$$
X+\sum_{t=\bar{t}}^{T-1} \pi_{t}\left(S_{t+1}-S_{t}\right)
$$

is linear in $X$ and $\Pi$ we have that the set of $X$ and $\Pi$ satisfying inequalities (1) forms a convex set in $X$ and $\Pi$. The projection of this set on the set of $X$ 's is also convex.

Let $\mathcal{X}_{\bar{t}}(\lambda)$ denote the set of all random variables measurable with respect to $\mathcal{N}_{\bar{t}}$ that satisfy the inequality

$$
\mathbb{E}^{\tilde{\mathbb{Y}}}[X] \geq \frac{f^{\mathbb{Q}}}{y_{0}}
$$


for all probability measures $\tilde{\mathbb{Y}}$, measures $\mathbb{Y}$ and probability measures $\mathbb{Q} \in \operatorname{co}\left\{\mathbb{P}^{i}\right.$, $i=1, \ldots, d\}$ such that

C1 $\frac{d \tilde{\mathbb{Y}}}{d \mathbb{Q}} \in \frac{1}{y_{0}}[1, \lambda]$

$\mathrm{C} 2 \mathrm{~S}$ is a martingale under the probability measure $\tilde{\mathbb{Y}} \equiv \frac{\mathbb{Y}}{y_{0}} \operatorname{over}\{\bar{t}, \bar{t}+1, \ldots, T\}$,

where $y_{0} \equiv \sum_{n \in \mathcal{N}_{T}} y_{n}$.

The main result of the paper is the following.

Theorem 1 For $t \in\{0,1, \ldots, T-1\}, \mathcal{G}_{t}(\lambda)=\mathcal{X}_{t}(\lambda)$.

We will use the linear programming duality theorem, Theorem 1.3.2 of Ben-Tal and Nemirovski (2001) to prove the theorem (see Appendix for a statement of the linear programming duality theorem). Let us first formulate the $\lambda$-acceptability as a particular linear programming problem with identically zero objective function, i.e., as a feasibility problem, referred to as $\mathrm{P}$, over variables $\pi_{n}$, and non-negative variables $x_{n}^{+}, x_{n}^{-}$.

$\min 0$

$$
\begin{gathered}
\text { s.t. } X_{\bar{t}}^{m}+\sum_{j \in \mathcal{A}(n, m) \backslash\{m\}} \pi_{t(a(j))}^{a(j)}\left(S_{t(j)}^{j}-S_{t(a(j))}^{a(j)}\right)=x_{n}^{+}-x_{n}^{-}, \quad \forall n \in \mathcal{N}_{T}, \forall m \in \mathcal{N}_{\bar{t}} \cap \mathcal{A}(n, 0), \\
\sum_{n \in \mathcal{N}_{T}} p_{n}^{i} x_{n}^{+}-\lambda \sum_{n \in \mathcal{N}_{T}} p_{n}^{i} x_{n}^{-} \geq f^{i}, \quad \forall i=1, \ldots, d, \\
x_{n}^{+}, x_{n}^{-} \geq 0, \quad \forall n \in \mathcal{N}_{T} .
\end{gathered}
$$

For ease of notation, let us denote the "leaf descendants" of an intermediate node $m$ in the tree by $\mathcal{L}(m)=\left\{n \in \mathcal{N}_{T} \mid m \in \mathcal{A}(n, 0)\right\}$. The dual problem (D) is the following maximization problem over the non-negative variables $\alpha^{i}, i=1, \ldots, d$, and the variables $y_{n}, n \in \mathcal{N}_{T}$.

$$
\begin{gathered}
\max \sum_{i=1}^{d} \alpha^{i} f^{i}-\sum_{m \in \mathcal{N}_{\bar{t}}} X_{\bar{t}}^{m}\left(\sum_{n \in \mathcal{L}(m)} y_{n}\right) \\
\text { s.t. } \sum_{\ell \in \mathcal{C}(m)} S_{t(\ell)}^{\ell}\left(\sum_{k \in \mathcal{C}(\ell)} y_{k}\right)=\left(\sum_{n \in \mathcal{L}(m)} y_{n}\right) S_{t}^{m}, \forall m \in \mathcal{N}_{t}, \forall \bar{t} \leq t \leq T-2 \\
\sum_{\ell \in \mathcal{C}(m)} S_{t(\ell)}^{\ell} y_{\ell}=\left(\sum_{n \in \mathcal{L}(m)} y_{n}\right) S_{T-1}^{m}, \forall m \in \mathcal{N}_{T-1}, \\
\sum_{i=1}^{d} \alpha^{i} p_{n}^{i} \leq y_{n} \leq \lambda \sum_{i=1}^{d} \alpha^{i} p_{n}^{i}, \forall n \in \mathcal{N}_{T} \\
\alpha^{i} \geq 0, \quad \forall i=1, \ldots, d .
\end{gathered}
$$


There are two preliminary observations. First, since $P$ is bounded (if it is feasible) and $D$ is trivially feasible (set all dual variables to zero), by linear programming duality theorem (see Appendix) we have that $P$ is feasible if and only if $D$ is bounded above. Second, while $y_{n}$ is defined only for leaf nodes, and it is not necessarily a probability distribution, it can be turned into a probability distribution $\tilde{y}_{n}$ after dividing all $y_{n}$ by $\sum_{n \in \mathcal{N}_{T}} y_{n}$ and, furthermore for each non-leaf (intermediate level) node in the tree we have, recursively,

$$
\tilde{y}_{n}=\sum_{m \in \mathcal{C}(n)} \tilde{y}_{m}, \quad \forall n \in \mathcal{N}_{t}, t=T-1, \ldots, 0 .
$$

After this scaling operation, we observe that the first set of constraints expresses the conditions that make the price process $S$ a martingale over $[\bar{t}, T]$ with respect to the tree filtration (cf. Definition 1).

Proof of Theorem 1 Now, let random variable $X$, measurable with respect to $\mathcal{N}_{t}$, be $\lambda$-acceptable, i.e., $X \in \mathcal{G}_{t}(\lambda)$. Hence, $P$ is feasible, which implies by weak duality of linear programming that $D$ is bounded above by zero. Therefore, whenever one can find $y_{n}, n \in \mathcal{N}_{T}$ which are not all zero satisfying the first set of constraints of the dual and $q_{n} \equiv \sum_{i=1}^{d} \alpha^{i} p_{n}^{i}$ where $\alpha^{i}$,s are not all zero and they add up to one, such that $1 \leq y_{n} / q_{n} \leq \lambda$ for all $n \in \mathcal{N}_{T}$, it is immediate to see that $\tilde{y}_{n}=y_{n} / \sum_{n \in \mathcal{N}_{T}} y_{n}$ makes $S$ a martingale over $[\bar{t}, T]$ after extending the $\tilde{y}_{n}$ to intermediate nodes as explained above. Obviously, the $y_{n}, n \in \mathcal{N}_{T}$ and $\alpha^{i}, i=1, \ldots, d$ so defined constitute a feasible solution for $D$. Since $D$ is bounded above by zero, we have

$$
\sum_{i=1}^{d} \alpha^{i} f^{i}-\sum_{m \in \mathcal{N}_{\bar{t}}} X_{\bar{t}}^{m}\left(\sum_{n \in \mathcal{L}(m)} y_{n}\right) \leq 0
$$

or, equivalently

$$
\sum_{m \in \mathcal{N}_{\bar{t}}} X_{\bar{t}}^{m}\left(\tilde{y}_{m}\right) \geq \frac{\sum_{i=1}^{d} \alpha^{i} f^{i}}{\sum_{n \in \mathcal{N}_{T}} y_{n}}
$$

Since this holds for any choice of $\alpha^{i}$ (not all zero) such that $q_{n}=\sum_{i=1}^{d} \alpha^{i} p_{n}^{i}$ for all $n \in \mathcal{N}_{T}$, we can rewrite the previous inequality

$$
\sum_{m \in \mathcal{N}_{\bar{t}}} X_{\bar{t}}^{m}\left(\tilde{y}_{m}\right) \geq \frac{f^{\mathbb{Q}}}{\sum_{n \in \mathcal{N}_{T}} y_{n}} .
$$

Thus, we have established that whenever one can find a probability measure $\tilde{\mathbb{Y}}$ and $\mathbb{Y}$ with $y_{0}$ and probability measure $Q \in \operatorname{co}\left\{\mathbb{P}^{i}, i=1, \ldots, d\right\}$ fulfilling conditions C1-C2 we have (2). This concludes the proof of $\mathcal{G}_{t}(\lambda) \subseteq \mathcal{X}_{t}(\lambda)$.

Now, for the converse, let us assume that random variable $X$ that is measurable with respect to $\mathcal{N}_{\bar{t}}$ belongs to $\mathcal{X}_{t}(\lambda)$. Then, for every probability measure $\tilde{\mathbb{Y}}$ and measure 
$\mathbb{Y}$ and probability measure $Q \in \operatorname{co}\left\{\mathbb{P}^{i}, i=1, \ldots, d\right\}$ fulfilling conditions $\mathrm{C} 1-\mathrm{C} 2$ we have that (2) holds for $X$. However, any measure $\mathbb{Y}$ along with probability measure $\mathbb{Q}$ that satisfy $\mathrm{C} 1-\mathrm{C} 2$ is a feasible solution to the following linear program D2

$$
\begin{array}{cc}
\max & \sum_{i=1}^{d} \alpha^{i} f^{i}-\sum_{m \in \mathcal{N}_{\bar{t}}} X_{\bar{t}}^{m}\left(\sum_{n \in \mathcal{L}(m)} y_{n}\right) \\
\text { s.t. } & \sum_{\ell \in \mathcal{C}(m)} S_{t(\ell)}^{\ell}\left(\sum_{k \in \mathcal{C}(\ell)} y_{k}\right)=\left(\sum_{n \in \mathcal{L}(m)} y_{n}\right) S_{t}^{m}, \quad \forall m \in \mathcal{N}_{t}, \forall \bar{t} \leq t \leq T-2 \\
\sum_{\ell \in \mathcal{C}(m)} S_{t(\ell)}^{\ell} y_{\ell}=\left(\sum_{n \in \mathcal{L}(m)} y_{n}\right) S_{T-1}^{m}, \quad \forall m \in \mathcal{N}_{T-1}, \\
\sum_{i=1}^{d} \alpha^{i} p_{n}^{i} \leq y_{n} \leq \lambda \sum_{i=1}^{d} \alpha^{i} p_{n}^{i}, \quad \forall n \in \mathcal{N}_{T} \\
\alpha^{i} \geq 0, \quad \forall i=1, \ldots, d \\
\sum_{i=1}^{d} \alpha^{i}=1 .
\end{array}
$$

which is dual to the linear program $\mathrm{P} 2$

$\min V$

$$
\begin{gathered}
\text { s.t. } X_{\bar{t}}^{m}+\sum_{j \in \mathcal{A}(n, m) \backslash\{m\}} \pi_{t(a(j))}^{a(j)}\left(S_{t(j)}^{j}-S_{t(a(j))}^{a(j)}\right)=x_{n}^{+}-x_{n}^{-}, \quad \forall n \in \mathcal{N}_{T}, \forall m \in \mathcal{N}_{\bar{t}} \cap \mathcal{A}(n, 0), \\
V+\sum_{n \in \mathcal{N}_{T}} p_{n}^{i} x_{n}^{+}-\lambda \sum_{n \in \mathcal{N}_{T}} p_{n}^{i} x_{n}^{-} \geq f^{i}, \quad \forall i=1, \ldots, d, \\
x_{n}^{+}, x_{n}^{-} \geq 0, \quad \forall n \in \mathcal{N}_{T} .
\end{gathered}
$$

over variables $V, \pi_{t}^{n}$, and non-negative variables $x_{n}^{+}, x_{n}^{-}$. Since under (11) any feasible solution to D2 leads to a non-positive objective function value, it holds that the optimal value in D2 is at most zero. This implies by the linear programming duality theorem that $\mathrm{P} 2$ is solvable with optimal value, $V^{*}$ say, which is also at most zero. This implies that $P$ is feasible, which in turn implies that $X \in \mathcal{G}_{t}(\lambda)$.

We shall explore special cases and consequences of the main result in subsequent sections.

\section{The Carr-Geman-Madan risk measure}

The Carr-Geman-Madan criterion is defined after specifying measures and associated floors as follows. For an investment opportunity to be acceptable the expected value at the fixed future date of the wealth process accumulated through trading should 
exceed the floor associated with each measure, and this should hold for every measure. Larsen et al. (2004), gave a characterization theorem for acceptable portfolio values à la Carr-Geman-Madan, akin to Theorem 1 above in a continuous-time semimartingale setting. We obtain a discrete time, finite state probability version of their result as a corollary of Theorem 1 .

For fixed $\bar{t} \in\{0,1, \ldots, T-1\} X$ measurable with respect to $\mathcal{N}_{\bar{t}}$, is acceptable at time $\bar{t}$ if there exists a trading strategy $\pi_{t}, t \in\{\bar{t}, \bar{t}+1, \ldots, T-1\}$ (see Larsen et al. 2004) such that

$$
\mathbb{E}^{\mathbb{P}^{i}}\left[X+\sum_{t=\bar{t}}^{T-1} \pi_{t}\left(S_{t+1}-S_{t}\right)\right] \geq f^{i} \quad \forall i=1, \ldots, d .
$$

Let $\mathcal{M}_{\bar{t}}$ be the set of all random variables $X$ measurable with respect to $\mathcal{N}_{\bar{t}}$ that are acceptable at time $\bar{t}$. It is again easy to verify that $\mathcal{M}_{\bar{t}}$ is a convex set.

Let $\mathcal{Q}_{\bar{t}}$ denote the set of all random variables measurable with respect to $\mathcal{N}_{\bar{t}}$ that satisfy the inequality

$$
\mathbb{E}^{\mathbb{Q}}[X] \geq f^{\mathbb{Q}}
$$

for all probability measures $\mathbb{Q}$ such that

CGM1 $\mathbb{Q} \in \operatorname{co}\left\{\mathbb{P}^{i}, i=1, \ldots, d\right\}$

CGM2 $S$ is a martingale under $\mathbb{Q}$ over $\{\bar{t}, \bar{t}+1, \ldots, T\}$.

Here $f^{\mathbb{Q}}$ is as defined previously.

Corollary 1 For $t \in\{0,1, \ldots, T-1\}, \mathcal{M}_{t}=\mathcal{Q}_{t}$.

The proof follows directly from Theorem 1 after taking $\lambda=1$, and consequently $y_{0}=1$.

\section{The Bernardo-Ledoit gain-loss risk constraint}

The Bernardo-Ledoit acceptability criterion can be viewed as follows. A risky investment is deemed acceptable if the ratio of expected positive part of portfolio values to their expected negative parts exceeds a certain allowable minimum $\lambda$. Equivalently, acceptability can be stated as the expected gains exceeding $\lambda$ times expected losses.

In our formulation (1) of $\lambda$-acceptability taking $f^{i}=0, i=1, \ldots, d$, we obtain the Bernardo-Ledoit gain-loss ratio criterion Bernardo and Ledoit (2000) extended to the presence of several trial measures. This extension allows to incorporate information about investor preferences by varying the choice of appropriately selected probability measures.

Choose $\lambda \geq 1$. We declare $X$, measurable with respect to $\mathcal{N}_{\bar{t}}$, BL-acceptable under trial measures at time $\bar{t}$ if there exists a trading strategy $\pi_{t}, t \in\{\bar{t}, \bar{t}+1, \ldots, T-1\}$ 
such that

$$
\begin{aligned}
\mathbb{E}^{\mathbb{P}^{i}} & {\left[\left(X+\sum_{t=\bar{t}}^{T-1} \pi_{t}\left(S_{t+1}-S_{t}\right)\right)_{+}\right] } \\
& -\lambda \mathbb{E}^{\mathbb{P}^{i}}\left[\left(X+\sum_{t=\bar{t}}^{T-1} \pi_{t}\left(S_{t+1}-S_{t}\right)\right)_{-}\right] \geq 0 \quad \forall i=1, \ldots, d .
\end{aligned}
$$

Let $\mathcal{B}_{t}(\lambda)$ be the set of all $\mathcal{N}_{\bar{t}}$ measurable random variables $X$ that are $\lambda$-BL-acceptable under trial measures at time $\bar{t}$.

Let $\mathcal{Z}_{t}(\lambda)$ denote the set of all $\mathcal{N}_{\bar{t}}$ measurable random variables $X$ that satisfy the inequality

$$
y_{0} \mathbb{E}^{\tilde{\mathbb{Y}}}[X] \geq 0
$$

for all probability measures $\tilde{\mathbb{Y}}$, measures $\mathbb{Y}$ and probability measures $\mathbb{Q} \in \operatorname{co}\left\{\mathbb{P}^{i}, i=\right.$ $1, \ldots, d\}$ such that

BL1 $\frac{d \tilde{Y}}{d \mathbb{Q}} \in \frac{1}{y_{0}}[1, \lambda]$

BL2 $S$ is a martingale under the probability measure $\tilde{\mathbb{Y}} \equiv \frac{\mathbb{Y}}{y_{0}}$ over $\{\bar{t}, \bar{t}+1, \ldots, T\}$.

Corollary 2 For $t \in\{0,1, \ldots, T-1\}, \mathcal{B}_{t}(\lambda)=\mathcal{Z}_{t}(\lambda)$.

If $d=1$, i.e., we only have a single scenario measure which is the original measure $\mathbb{P}$ itself, then we are in the situation developed in the original Bernardo and Ledoit (2000). In this case, let us denote the acceptable set at time $t$ for a single measure by $\mathcal{B}_{t}^{s}(\lambda)$, and let $\mathcal{Z}_{\bar{t}}^{s}(\lambda)$ denote the set of all random variables $X$ measurable with respect to $\mathcal{N}_{\bar{t}}$ that satisfy the inequality

$$
y_{0} \mathbb{E}^{\tilde{\mathbb{Y}}}[X] \geq 0
$$

for all probability measures $\tilde{\mathbb{Y}}$ and measures $\mathbb{Y}$ fulfilling

BL1 $\frac{d \tilde{\mathbb{Y}}}{d \mathbb{P}} \in \frac{1}{y_{0}}[1, \lambda]$

BL2 $S$ is a martingale under the probability measure $\tilde{\mathbb{Y}} \equiv \frac{\mathbb{Y}}{y_{0}}$ over $\{\bar{t}, \bar{t}+1, \ldots, T\}$.

Corollary 3 For $t \in\{0,1, \ldots, T-1\}, \mathcal{B}_{t}^{s}(\lambda)=\mathcal{Z}_{t}^{s}(\lambda)$.

\section{Acceptance today and associated optimization problems}

A useful ramification of our results so far is to consider acceptability at time $t=0$ for pricing contingent claims. 
We declare $x \in \mathbb{R} \lambda$-acceptable at time $t=0$ if there exists a trading strategy $\Pi$ such that

$$
\begin{aligned}
& x+ \mathbb{E}^{\mathbb{P}^{i}}\left[\left(\sum_{t=0}^{T-1} \pi_{t}\left(S_{t+1}-S_{t}\right)\right)_{+}\right] \\
&-\lambda \mathbb{E}^{\mathbb{P}^{i}}\left[\left(\sum_{t=0}^{T-1} \pi_{t}\left(S_{t+1}-S_{t}\right)\right)_{-}\right] \geq f^{i} \quad \forall i=1, \ldots, d .
\end{aligned}
$$

Let $\mathcal{G}_{0}(\lambda)$ be the set of all $x \in \mathbb{R}$ that are $\lambda$-acceptable at time $t=0$.

As a result of Theorem 1 , the $\lambda$-acceptance set $\mathcal{G}_{0}(\lambda)$ at time $t=0$ is characterized as follows. Let $\mathcal{X}_{0}(\lambda)$ denote the set of real numbers $x$ satisfying the inequality

$$
x \geq \frac{f^{\mathbb{Q}}}{y_{0}}
$$

for all measures $\mathbb{Y}$ and probability measures $\tilde{\mathbb{Y}}$, and $\mathbb{Q} \in \operatorname{co}\left\{\mathbb{P}^{i}, i=1, \ldots, d\right\}$ satisfying $(\mathrm{C} 1)-(\mathrm{C} 2)$.

Then, as a corollary to Theorem 1 , we have that $\mathcal{G}_{0}(\lambda)=\mathcal{X}_{0}(\lambda)$.

It is immediately seen that the lower limit for $\lambda$-acceptable initial positions at time 0 can also be viewed as the optimal value, $\xi(0)$, of the optimization problem, referred to as $D_{\lambda}$

$$
\sup \left\{\frac{\sum_{i=1}^{d} \alpha^{i} f^{i}}{\sum_{n \in \mathcal{N}_{T}} y_{n}} \mid(\tilde{\mathbb{Y}}, \mathbb{Y}, \alpha) \in \mathcal{M}\right\}
$$

where the set $\mathcal{M}$ is the set of all $(\tilde{\mathbb{Y}}, \mathbb{Y}, \alpha)$ such that $\alpha^{i} \geq 0, \sum_{i=1}^{d} \alpha^{i}=1$ satisfies $\frac{d \tilde{\mathbb{Y}}}{d \mathbb{Q}} \in \frac{1}{y_{0}}[1, \lambda]$ for $\mathbb{Q}=\sum^{c} \alpha^{i} \mathbb{P}^{i}$ and where $S$ is a martingale under $\tilde{\mathbb{Y}} \equiv \frac{\mathbb{Y}}{\sum_{n \in \mathcal{N}_{T} y_{n}}}$ over $\{0,1, \ldots, T\}$. The optimization problem $D_{\lambda}$ serves to optimize the objective function over the non-trivial feasible solutions to $D$.

Based on $D$, we can express this optimization problem $D_{\lambda}$ as

$$
\begin{array}{ll}
\max & \frac{\sum_{i=1}^{d} \alpha^{i} f^{i}}{\sum_{n \in \mathcal{N}_{T}} y_{n}} \\
\text { s.t. } \quad y_{m}=\sum_{n \in \mathcal{C}(m)} y_{n}, \quad \forall m \in \mathcal{N}_{t}, 0 \leq t \leq T-1 \\
\tilde{y}_{m} S_{t}^{m}=\sum_{n \in \mathcal{C}(m)} \tilde{y}_{n} S_{t(n)}^{n}, \quad \forall m \in \mathcal{N}_{t}, \quad 0 \leq t \leq T-1 \\
q_{n}=\sum_{i=1}^{d} \alpha^{i} p_{n}^{i}, \quad \forall n \in \mathcal{N}_{T}
\end{array}
$$




$$
\begin{aligned}
& \tilde{y}_{n}=y_{n} / \sum_{n \in \mathcal{N}_{T}} y_{n}, \quad \forall n \\
& \tilde{y}_{m}=\sum_{n \in \mathcal{C}(m)} \tilde{y}_{n}, \quad \forall m \in \mathcal{N}_{t}, t \leq T-1 \\
& q_{n} \leq y_{n} \leq \lambda q_{n} \forall n \in \mathcal{N}_{T} . \\
& \alpha^{i} \geq 0, \quad \forall i=1, \ldots, d \\
& \sum_{i=1}^{d} \alpha^{i}=1 .
\end{aligned}
$$

The above problem can be simplified to a linear-fractional problem (which can be processed numerically in a routine manner by modern software) since we can get rid of the variables $\tilde{y}$ :

$$
\begin{array}{ll}
\max & \frac{\sum_{i=1}^{d} \alpha^{i} f^{i}}{\sum_{n \in \mathcal{N}_{T}} y_{n}} \\
\text { s.t. } \quad y_{m}=\sum_{n \in \mathcal{C}(m)} y_{n}, \quad \forall m \in \mathcal{N}_{t}, 0 \leq t \leq T-1 \\
y_{m} S_{t}^{m}=\sum_{n \in \mathcal{C}(m)} y_{n} S_{t(n)}^{n}, \quad \forall m \in \mathcal{N}_{t}, \quad 0 \leq t \leq T-1 \\
q_{n}=\sum_{i=1}^{d} \alpha^{i} p_{n}^{i}, \quad \forall n \in \mathcal{N}_{T} \\
q_{n} \leq y_{n} \leq \lambda q_{n} \quad \forall n \in \mathcal{N}_{T} . \\
\alpha^{i} \geq 0, \forall i=1, \ldots, d \\
\sum_{i=1}^{d} \alpha^{i}=1 .
\end{array}
$$

Notice that the feasible set of the above problem coincides with that of D2. A further simplification by defining a new set of variables $\tilde{q}_{n}$, for $n \in \mathcal{N}$, and $\tilde{\alpha}^{i}$, for $i=1, \ldots, d$ using the relations

$$
\sum_{n \in \mathcal{N}_{T}} y_{n}=y_{0}=\frac{1}{\sum_{i=1}^{d} \tilde{\alpha}^{i}}, \quad \tilde{q}_{n}=y_{n} / y_{0}, n \in \mathcal{N}, \tilde{\alpha}_{i}=\gamma \alpha_{i}, i=1, \ldots, d
$$

results in the equivalent linear programming problem:

$$
\begin{array}{ll}
\max & \sum_{i=1}^{d} \tilde{\alpha}^{i} f^{i} \\
\text { s.t. } & \tilde{q}_{m} S_{t}^{m}=\sum_{n \in \mathcal{C}(m)} \tilde{q}_{n} S_{t(n)}^{n}, \quad \forall m \in \mathcal{N}_{t}, \quad 0 \leq t \leq T-1
\end{array}
$$




$$
\begin{aligned}
& \tilde{q}_{0}=1 \\
& \sum_{i=1}^{d} \tilde{\alpha}^{i} p_{n}^{i} \leq \tilde{q}_{n} \leq \lambda \sum_{i=1}^{d} \tilde{\alpha}^{i} p_{n}^{i} \quad \forall n \in \mathcal{N}_{T} . \\
& \tilde{\alpha}^{i} \geq 0, \quad \forall i=1, \ldots, d .
\end{aligned}
$$

Therefore, we managed to transform $D_{\lambda}$ into an equivalent linear programming problem.

For the Carr-Geman-Madan risk constraint we simply take $\lambda=1$ and obtain the linear programming problem DCGM

$$
\begin{aligned}
& \max \sum_{i=1}^{d} \alpha^{i} f^{i} \\
& \text { s.t. } \quad q_{m}=\sum_{n \in \mathcal{C}(m)} q_{n}, \forall m \in \mathcal{N}_{t}, 0 \leq t \leq T-1 \\
& q_{m} S_{t}^{m}=\sum_{n \in \mathcal{C}(m)} q_{n} S_{t(n)}^{n}, \forall m \in \mathcal{N}_{t}, 0 \leq t \leq T-1 \\
& q_{n}=\sum_{i=1}^{d} \alpha^{i} p_{n}^{i}, \forall n \in \mathcal{N}_{T} \\
& \alpha^{i} \geq 0, \forall i=1, \ldots, d \\
& \sum_{i=1}^{d} \alpha^{i}=1 .
\end{aligned}
$$

For the Bernardo-Ledoit gain-loss risk constraint under trial measures we obtain the (linear) feasibility problem DBL

$$
\begin{aligned}
& \max 0 \\
& \text { s.t. } y_{m}=\sum_{n \in \mathcal{C}(m)} y_{n}, \forall m \in \mathcal{N}_{t}, 0 \leq t \leq T-1 \\
& y_{m} S_{t}^{m}=\sum_{n \in \mathcal{C}(m)} y_{n} S_{t(n)}^{n}, \forall m \in \mathcal{N}_{t}, 0 \leq t \leq T-1 \\
& q_{n}=\sum_{i=1}^{d} \alpha^{i} p_{n}^{i}, \forall n \in \mathcal{N}_{T} \\
& q_{n} \leq y_{n} \leq \lambda q_{n} \forall n \in \mathcal{N}_{T} \\
& \alpha^{i} \geq 0, \forall i=1, \ldots, d \\
& \sum_{i=1}^{d} \alpha^{i}=1 \text {. }
\end{aligned}
$$


We shall now utilize the results of this section in pricing of European contingent claims using gain-loss criterion and its variants.

\section{Contingent claims and pricing}

Assume that a European contingent claim $C$ with pay-off $C_{T}=\left\{C_{n}\right\}_{n \in \mathcal{N}_{T}}$ is available. The writer of the claim, who is $\beta$ units short of the claim, is interested in computing the initial outlay $X$ at time $t \in\{0,1, \ldots, T-1\}$ that will replicate the claim through trading until time $T$ in the sense that he/she seeks a trading strategy $\pi$ such that

$$
\begin{aligned}
\mathbb{E}^{\mathbb{P}^{i}} & {\left[\left(X+\sum_{t=\bar{t}}^{T-1} \pi_{t}\left(S_{t+1}-S_{t}\right)-\beta C\right)_{+}\right] } \\
& -\lambda \mathbb{E}^{\mathbb{P}^{i}}\left[\left(X+\sum_{t=\bar{t}}^{T-1} \pi_{t}\left(S_{t+1}-S_{t}\right)-\beta C\right)_{-}\right] \geq f^{i} \quad \forall i=1, \ldots, d .
\end{aligned}
$$

Let $\mathcal{G}_{\bar{t}}^{C}(\lambda)$ be the set of all random variables $X$ measurable with respect to $\mathcal{N}_{\bar{t}}$ such that there exists a trading strategy $\Pi$ satisfying the above system of linear inequalities time at $\bar{t}$.

Let $\mathcal{X}_{\bar{t}}^{C}(\lambda)$ denote the set of all random variables measurable with respect to $\mathcal{N}_{\bar{t}}$ that satisfy the inequality

$$
\mathbb{E}^{\tilde{\mathbb{Y}}}[X] \geq \frac{f^{\mathbb{Q}}+\beta \mathbb{E}^{\tilde{Y}}\left[C_{T}\right]}{y_{0}}
$$

for all measures $\mathbb{Y}$ and probability measures $\tilde{Y}$ and $\mathbb{Q} \in \operatorname{co}\left\{\mathbb{P}^{i}, i=1, \ldots, d\right\}$ satisfying conditions $\mathrm{C} 1-\mathrm{C} 2$.

The following result is proved exactly as Theorem 1, hence the proof is left as an exercise.

Corollary 4 For $t \in\{0,1, \ldots, T-1\}, \mathcal{G}_{t}^{C}(\lambda)=\mathcal{X}_{t}^{C}(\lambda)$.

This result can again be specialized to Carr-Geman-Madan and Bernardo-Ledoit risk constraints as in Sects. 4 and 5, respectively.

The lower limit for $\lambda$-acceptable initial positions at time 0 in the presence of a short position $\beta$ in the claim $C$ can also be viewed as the optimal value, $\xi(\beta)$, of the optimization problem, referred to as $D_{\lambda}^{C}$

$$
\sup \left\{\frac{\sum_{i=1}^{d} \alpha^{i} f^{i}+\beta \mathbb{E}^{\tilde{\mathbb{Y}}}\left[C_{T}\right]}{\sum_{n \in \mathcal{N}_{T}} y_{n}} \mid(\tilde{\mathbb{Y}}, \mathbb{Y}, \alpha) \in \mathcal{M}\right\}
$$


Based on $D$, we can rewrite this optimization problem $D_{\lambda}^{C}$ as

$$
\begin{aligned}
& \max \frac{\sum_{i=1}^{d} \alpha^{i} f^{i}+\beta \mathbb{E}^{\tilde{\mathbb{Y}}}\left[C_{T}\right]}{\sum_{n \in \mathcal{N}_{T}} y_{n}} \\
& \text { s.t. } \quad y_{m}=\sum_{n \in \mathcal{C}(m)} y_{n}, \quad \forall m \in \mathcal{N}_{t}, 0 \leq t \leq T-1 \\
& \tilde{y}_{m} S_{t}^{m}=\sum_{n \in \mathcal{C}(m)} \tilde{y}_{n} S_{t(n)}^{n}, \quad \forall m \in \mathcal{N}_{t}, 0 \leq t \leq T-1 \\
& q_{n}=\sum_{i=1}^{d} \alpha^{i} p_{n}^{i}, \quad \forall n \in \mathcal{N}_{T} \\
& \tilde{y}_{n}=y_{n} / \sum_{n \in \mathcal{N}_{T}} y_{n}, \quad \forall n \\
& \tilde{y}_{m}=\sum_{n \in \mathcal{C}(m)} \tilde{y}_{n}, \quad \forall m \in \mathcal{N}_{t}, t \leq T-1 \\
& q_{n} \leq y_{n} \leq \lambda q_{n} \quad \forall n \in \mathcal{N}_{T} . \\
& \alpha^{i} \geq 0, \quad \forall i=1, \ldots, d \\
& \sum_{i=1}^{d} \alpha^{i}=1,
\end{aligned}
$$

or, equivalently as,

$$
\begin{array}{ll}
\max & \frac{\sum_{i=1}^{d} \alpha^{i} f^{i}+\beta \sum_{n \in \mathcal{N}_{T}} y_{n} C_{n}}{\sum_{n \in \mathcal{N}_{T}} y_{n}} \\
\text { s.t. } \quad y_{m}=\sum_{n \in \mathcal{C}(m)} y_{n}, \quad \forall m \in \mathcal{N}_{t}, 0 \leq t \leq T-1 \\
y_{m} S_{t}^{m}=\sum_{n \in \mathcal{C}(m)} y_{n} S_{t(n)}^{n}, \quad \forall m \in \mathcal{N}_{t}, 0 \leq t \leq T-1 \\
q_{n}=\sum_{i=1}^{d} \alpha^{i} p_{n}^{i}, \quad \forall n \in \mathcal{N}_{T} \\
q_{n} \leq y_{n} \leq \lambda q_{n} \quad \forall n \in \mathcal{N}_{T} . \\
\alpha^{i} \geq 0, \quad \forall i=1, \ldots, d \\
\sum_{i=1}^{d} \alpha^{i}=1 .
\end{array}
$$


and, finally passing to the equivalent linear programming problem as $D_{\text {price }}$

$$
\begin{aligned}
& \max \sum_{i=1}^{d} \tilde{\alpha}^{i} f^{i}+\beta \mathbb{E}^{\mathbb{\mathbb { Q }}}\left[C_{T}\right] \\
& \text { s.t. } \quad \tilde{q}_{m} S_{t}^{m}=\sum_{n \in \mathcal{C}(m)} \tilde{q}_{n} S_{t(n)}^{n}, \quad \forall m \in \mathcal{N}_{t}, \quad 0 \leq t \leq T-1 \\
& \tilde{q}_{0}=1 \\
& \quad \sum_{i=1}^{d} \tilde{\alpha}^{i} p_{n}^{i} \leq \tilde{q}_{n} \leq \lambda \sum_{i=1}^{d} \tilde{\alpha}^{i} p_{n}^{i} \quad \forall n \in \mathcal{N}_{T} . \\
& \tilde{\alpha}^{i} \geq 0, \quad \forall i=1, \ldots, d .
\end{aligned}
$$

For the Carr-Geman-Madan risk constraint we again take $\lambda=1$ and $y_{0}=1$ to obtain the linear programming problem with objective function

$$
\max _{\mathbb{Q}} \sum_{i=1}^{d} \alpha^{i} f^{i}+\beta \mathbb{E}^{\mathbb{Q}}\left[C_{T}\right]
$$

over the constraints of problem DCGM. For the Bernardo-Ledoit gain-loss risk constraint under trial measures we obtain the linear-fractional programming problem with objective function

$$
\max _{y} \beta \frac{\sum_{n \in \mathcal{N}_{T}} y_{n} C_{n}}{\sum_{n \in \mathcal{N}_{T}} y_{n}}
$$

subject to the constraints of DBL. However, this problem can be equivalently posed as the linear programming problem:

$$
\begin{array}{ll}
\max & \beta \mathbb{E}^{\tilde{\mathbb{Q}}}\left[C_{T}\right] \\
\text { s.t. } & \tilde{q}_{m} S_{t}^{m}=\sum_{n \in \mathcal{C}(m)} \tilde{q}_{n} S_{t(n)}^{n}, \quad \forall m \in \mathcal{N}_{t}, 0 \leq t \leq T-1 \quad \tilde{q}_{0}=1 \\
& \sum_{i=1}^{d} \tilde{\alpha}^{i} p_{n}^{i} \leq \tilde{q}_{n} \leq \lambda \sum_{i=1}^{d} \tilde{\alpha}^{i} p_{n}^{i} \quad \forall n \in \mathcal{N}_{T} . \\
& \tilde{\alpha}^{i} \geq 0, \quad \forall i=1, \ldots, d .
\end{array}
$$

For an investor who is long $\beta$ units of the claim, one can repeat the above development verbatim by replacing all occurrences of $\beta$ by $-\beta$.

Following Larsen et al. (2004) the value (price) of a claim to the gain loss ratio risk optimizing seller is given by the quantity $p(1)=\xi(1)-\xi(0)$ since the agent 
who is short the claim will need additional initial endowment $\xi(1)-\xi(0)$. This seller price $p(1)$ is dominated by the no-arbitrage seller's price $\sup \left\{\mathbb{E}^{\tilde{\mathbb{Y}}}\left[C_{T}\right] \mid \tilde{\mathbb{Y}} \in \mathrm{Q}\right\}$ by the following reasoning. We observe that

$$
\begin{aligned}
\xi(1) & =\sup \left\{\frac{\sum_{i=1}^{d} \alpha^{i} f^{i}+\mathbb{E}^{\tilde{\mathbb{Y}}}\left[C_{T}\right]}{\sum_{n \in \mathcal{N}_{T}} y_{n}} \mid(\tilde{\mathbb{Y}}, \mathbb{Y}, \alpha) \in \mathcal{M}\right\} \\
& \leq \xi(0)+\sup \left\{\frac{\mathbb{E}^{\tilde{\mathbb{Y}}}\left[C_{T}\right]}{\sum_{n \in \mathcal{N}_{T}} y_{n}} \mid(\tilde{\mathbb{Y}}, \mathbb{Y}, \alpha) \in \mathcal{M}\right\} .
\end{aligned}
$$

Since we have

$$
\sup \left\{\frac{\mathbb{E}^{\tilde{\mathbb{Y}}}\left[C_{T}\right]}{\sum_{n \in \mathcal{N}_{T}} y_{n}} \mid(\tilde{\mathbb{Y}}, \mathbb{Y}, \alpha) \in \mathcal{M}\right\} \leq \sup \left\{\mathbb{E}^{\tilde{\mathbb{Y}}}\left[C_{T}\right] \mid \tilde{\mathbb{Y}} \in \mathbf{Q}\right\}
$$

the assertion follows. Similarly, we have that the investor who is long the claim $C$, the additional initial capital requirement is $\xi(-1)$, which leads to a buyer's price of $-p(-1)=\xi(0)-\xi(-1)$. This price $-p(-1)$ is bounded below by the price for the no-arbitrage buyer: $\inf \left\{\mathbb{E}^{\tilde{Y}}\left[C_{T}\right] \mid \tilde{\mathbb{Y}} \in Q\right\}$. To see this, observe that

$$
\begin{aligned}
\xi(-1) & =\sup \left\{\frac{\sum_{i=1}^{d} \alpha^{i} f^{i}-\mathbb{E}^{\tilde{\mathbb{Y}}}\left[C_{T}\right]}{\sum_{n \in \mathcal{N}_{T}} y_{n}} \mid(\tilde{\mathbb{Y}}, \mathbb{Y}, \alpha) \in \mathcal{M}\right\} \\
& \geq \inf \left\{-\frac{\sum_{i=1}^{d} \alpha^{i} f^{i}}{\sum_{n \in \mathcal{N}_{T}} y_{n}} \mid(\tilde{\mathbb{Y}}, \mathbb{Y}, \alpha) \in \mathcal{M}\right\}+\inf \left\{\frac{\mathbb{E}^{\tilde{\mathbb{Y}}}\left[C_{T}\right]}{\sum_{n \in \mathcal{N}_{T}} y_{n}} \mid(\tilde{\mathbb{Y}}, \mathbb{Y}, \alpha) \in \mathcal{M}\right\} .
\end{aligned}
$$

Since we have

$$
\inf \left\{\frac{\mathbb{E}^{\tilde{\mathbb{Y}}}\left[C_{T}\right]}{\sum_{n \in \mathcal{N}_{T}} y_{n}} \mid(\tilde{\mathbb{Y}}, \mathbb{Y}, \alpha) \in \mathcal{M}\right\} \geq \inf \left\{\mathbb{E}^{\tilde{\mathbb{Y}}}\left[C_{T}\right] \mid \tilde{\mathbb{Y}} \in \mathbf{Q}\right\}
$$

the desired inequality follows. These two assertions show that the lower and upper prices obtained using the gain-loss criterion are tighter than the no-arbitrage price bounds.

\section{Option pricing bounds under trial measures}

In this section, inspired by a numerical example in Bernardo and Ledoit (2000), and Longarela (2002), we illustrate the use of the results developed in the paper for computing price bounds on a European call option written on a stock in a Black-Scholes economy with alternative volatility specifications, first under the assumption that intermediate trading between the present and the expiry date of the option is not allowed, and then, in the absence of this assumption. Merton (1973) (see also Rubinstein 1976) 
showed that in a Black-Scholes setting without intermediate trading an appropriately chosen benchmark pricing kernel (risk-neutral probability measure) implies an option price equal to the Black-Scholes price. We shall see below that our price bounds converge to the Black-Scholes price corresponding to one of the volatility specifications as the loss aversion parameter $\lambda$ goes down to one.

Assume we have a stock and a bond, and we are trying to price a European call option written on a stock, with strike price equal to 100, and 1 year to expiration. The 1 year continuously compounded rate of return of the bond is $4.88 \%$. We will take the viewpoint of the investor whose confidence in the volatility data in a Black-Scholes setting is limited, and will compute price bounds under this setting. For further details about this example, we refer the reader to Section 3 of Longarela (2002) and Section V of Bernardo and Ledoit (2000).

Consider a discretization of the stock price at expiration of the option. We take as the possible realizations of the stock price the values $S_{1}=41, S_{2}=42, \ldots, S_{120}=160$. Hence, the market is grossly incomplete. The option pay-off at expiration $C_{\ell}$ takes the value 0 for $\ell=1, \ldots, 60$ and then evolves as $C_{61}=1, C_{62}=2, \ldots, C_{120}=60$. Assume the stock price is currently equal to 95 . The no-arbitrage bounds for this incomplete market example are quite far apart: 0 and 28.21. We use the Black-Scholes risk-neutral probabilities as scenario measures under two different values of $\sigma$, namely $\sigma^{1}=0.1409$ (the value used in the example in Bernardo and Ledoit (2000)) and $\sigma^{2}=$ 0.2. Hence we compute $p_{1}^{i}=\operatorname{Pr}\left\{\gamma^{i} \leq \ln 41.5\right\}, p_{2}^{i}=\operatorname{Pr}\left\{\gamma^{i} \in(\ln 41.5, \ln 42.5]\right\}$, and so on until $p_{119}^{i}=\operatorname{Pr}\left\{\gamma^{i} \in(\ln 158.5, \ln 159.5]\right\}$ and $p_{120}^{i}=\operatorname{Pr}\{\gamma>\ln 159.5\}$, where $\gamma^{i}$ are normally distributed random variables with mean $\ln 95+0.0488-\frac{\left(\sigma^{i}\right)^{2}}{2}$ and standard deviation $\sigma^{i}$ for $i=1,2$. The floors $f^{1}$ and $f^{2}$ are equal to zero. In other words, the probability measures $\mathbb{P}^{1}$ and $\mathbb{P}^{2}$ are "valuation measures" in the terminology of Carr-Madan-Geman. Furthermore, we use a third measure $\mathbb{P}^{3}$ as stress measure, and pick $p_{n}^{3}=1 / 60$ for $n=1, \ldots, 60$, and $f^{3}=-0.001$.

We solve the resulting pricing problems $D_{\text {price }}$ for both the seller and buyer and plot the resulting values, $p(1)$ and $-p(-1)$ of the previous section, as a function of $\lambda$ in Fig. 1. Our first observation is that the price bounds are very reasonable compared to the no-arbitrage bounds which were far apart. A second observation is that the seller bounds show a steeper decrease as $\lambda$ approaches one while the buyer bounds change almost linearly. The piecewise linear shape of the seller price curve stems from the following observed fact. For values of $\lambda$ over a certain threshold $(\lambda>1.009)$ in our chosen numerical example, the stress measure $\mathbb{P}^{3}$ plays a role in computing the price bound (i.e., the corresponding $\alpha^{3}$ is positive in the optimal solution, which implies that the risk constraint is tight since the investor is increasingly more loss averse as $\lambda$ is larger), but as $\lambda$ is decreased further, the emphasis switches to probability measure $\mathbb{P}^{1}$, i.e., the benchmark risk-neutral Black-Scholes measure corresponding to $\sigma^{1}$, and the seller price converges to the associated continuous-time Black-Scholes price of 5.22. The behavior of the variables $\alpha^{i}, i=1,2,3$ is exhibited in Fig. 2 for values of $\lambda$ close to one.

On the other hand, for the entire range of $\lambda$ exhibited in the figures, the buyer price is dominated by $\mathbb{P}^{1}$, and it converges to the continuous-time Black-Scholes price of 5.22 as in Bernardo and Ledoit (2000). 


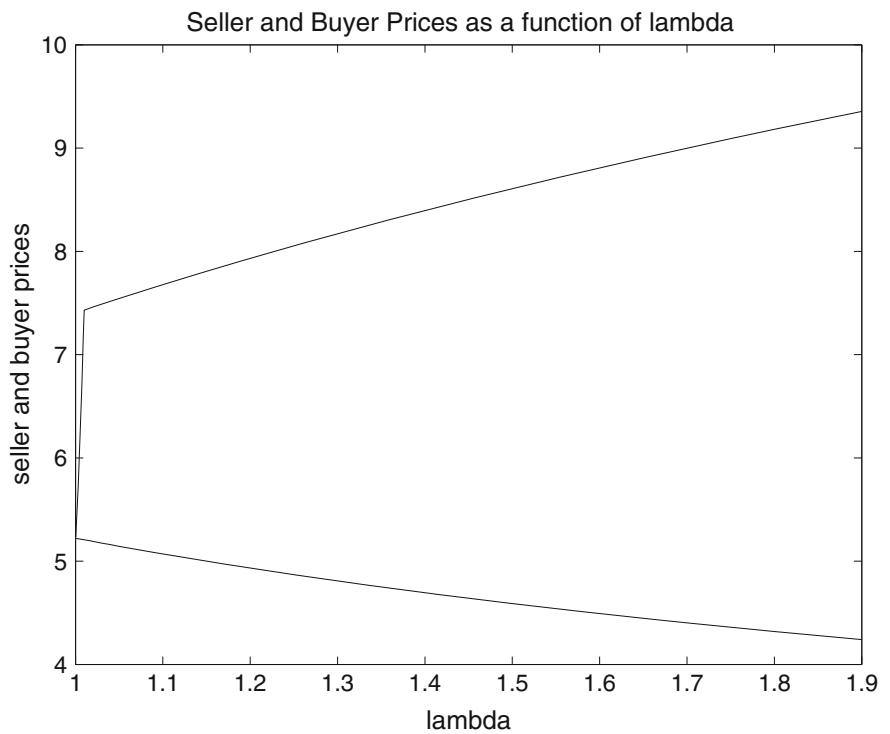

Fig. 1 Bounds on call option price where the initial price of the underlying is equal to 95. The option expires in 1 year and has strike $K=100$, the 1 year continuously compounded risk free rate of return is $4.88 \%$, and and the standard deviation of the continuously compounded rate of return on the stock is taken to $\sigma_{1}=0.1409$, and $\sigma_{2}=0.2$ per year, respectively. The probability distribution $\mathbb{P}^{3}$ gives equal weight to the first 60 states of nature, and the associated floor is equal to -0.001 . The upper curve represents the seller price bounds while the lower curve represents the buyer price bounds

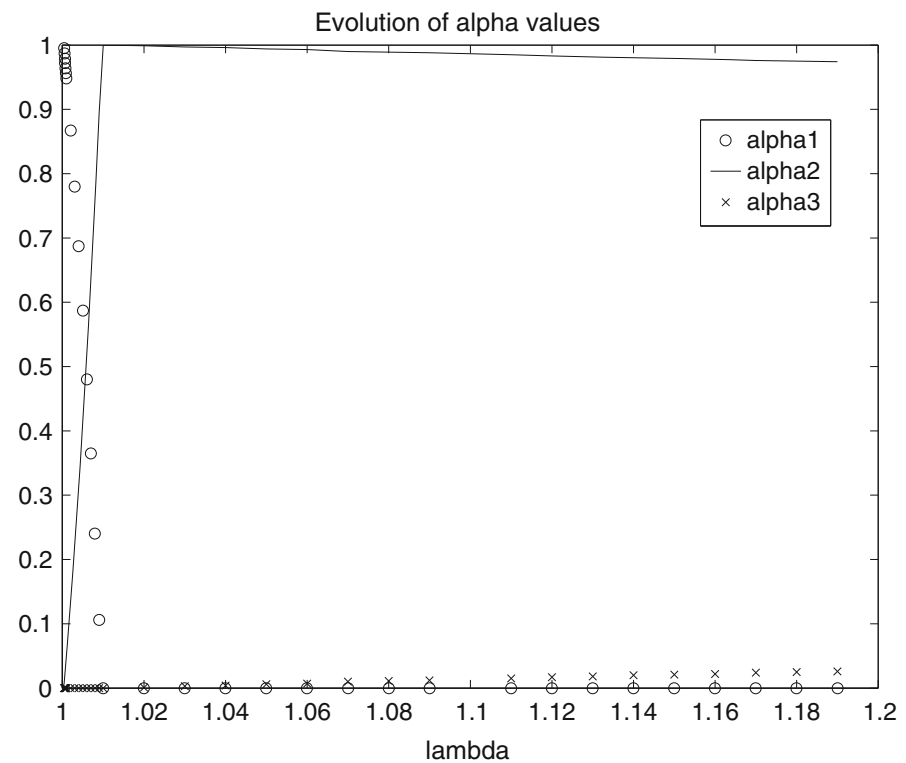

Fig. 2 Behavior of $\alpha^{i}$ s 


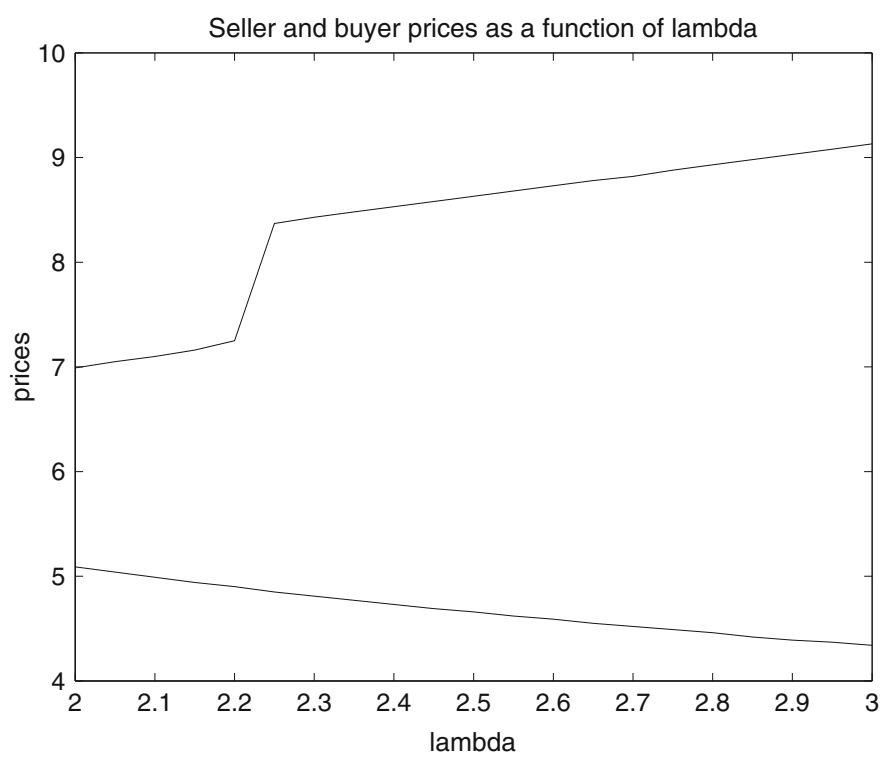

Fig. 3 Bounds on call option price where the initial price of the underlying is equal to 95 with intermediate trading after six months and parameters identical to those of Fig. 1. The upper curve represents the seller price bounds while the lower curve represents the buyer price bounds

In our second example, we allow trading in the bond and in the underlying after six months. We construct the following scenario tree. At the intermediate trading point the stock can take the following eleven different values $(44,55,66,77,88,99,110,121,132,143,154)$ with probabilities $p_{1}^{i}=\operatorname{Pr}\left\{\gamma^{i} \leq\right.$ $\ln 44.5\}, p_{2}^{i}=\operatorname{Pr}\left\{\gamma^{i} \in(\ln 44.5, \ln 55.5]\right\}$, and so on until $p_{10}^{i}=\operatorname{Pr}\left\{\gamma^{i} \in\right.$ $(\ln 132.5, \ln 143.5]\}$ and $p_{120}^{i}=\operatorname{Pr}\{\gamma>\ln 143.5\}$, where $\gamma^{i}$ are normally distributed random variables with mean $\ln 95+\frac{1}{2}\left(0.0488-\frac{\left(\sigma^{i}\right)^{2}}{2}\right)$ and standard deviation $\sigma^{i} * \sqrt{0.5}$ for $i=1,2$. Each of these eleven nodes branch out to eleven further nodes with values ranging from 41 to 161 with a unit increment, i.e. 121 nodes. We calculate the conditional probabilities again and obtain the probability distribution of the 121 scenario paths. The no-arbitrage bounds for this option are 1.17 and 26.7, respectively. The stress measure is chosen as $\mathbb{P}^{3}$ along with its floor $f^{3}$ exactly as in the previous example.

In Fig. 3, we plot the upper and lower bounds obtained in the range of values of $\lambda$ from two to three. Below $\lambda=2$, the problems become infeasible. In this example one cannot decrease $\lambda$ to one. The behavior of multipliers $\alpha$ is similar to the case of the previous example (so, we omit the supporting plot) in that the stress measure $\mathbb{P}^{3}$ plays a role in pricing the option through a positive optimal value of $\alpha^{3}$ (along with measure $\mathbb{P}^{1}$ ) while measure $\mathbb{P}^{2}$ becomes increasingly insignificant, as $\lambda$ approaches the critical value of 2 , and the investor's risk appetite increases. For values of $\lambda$ in the range from 10 to 200 , say where the investor loss aversion is significant, measure $\mathbb{P}^{1}$ dominates. 


\section{Conclusions and future research}

In this paper, we characterized the set of random variables that can be traded to acceptability defined as a gain-loss criterion exceeding certain floors associated with different measures. Our result, obtained in a discrete time finite probability setting, allowed to obtain as special cases the acceptability criterion of Larsen et al. (2004) (developed in continuous time) and that of Bernardo and Ledoit (2000). The result was obtained through linear programming duality, and leads to linear optimization problems for pricing contingent claims, which are easily processed numerically. The results were illustrated on examples from option pricing with and without intermediate trading. An extension to the continuous trading setting as in Larsen et al. (2004) is a topic of current study.

\section{Appendix}

For the reader's convenience we reproduce the linear programming duality theorem, Theorem 1.3.2 from Ben-Tal and Nemirovski (2001, p. 18).

Theorem 2 Consider the linear program

$$
\min _{x}\left\{c^{T} x \mid A x \geq b\right\}
$$

along with its dual

$$
\max _{y}\left\{b^{T} y \mid A^{T} y=c\right\} .
$$

Then

1. the duality is symmetric: the problem dual to dual is equivalent to the primal;

2. (weak duality) the value of the dual objective at every dual feasible solution is less than or equal to the value of primal objective at every primal feasible solution;

3. the following properties are equivalent to each other;

(i) the primal is feasible and bounded below,

(ii) the dual is feasible and bounded above,

(iii) the primal is solvable,

(iv) the the dual is solvable,

(v) both primal and dual are feasible.

Whenever $(i) \equiv(i i) \equiv(i i i) \equiv(i v) \equiv(v)$ is the case, the optimal values of the primal and the dual problems are equal to each other.

\section{References}

Barberis N, Huand M, Santos T (2001) Prospect theory and asset prices. Q J Econ CXVI(1):1-53

Ben-Tal A, Nemirovski AS (2001) Lectures on modern convex optimization: analysis, algorithms and engineering applications. MPS-SIAM Series on Optimization. SIAM, Philadelphia 
Bernardo AE, Ledoit O (2000) Gain, loss and asset pricing. J Polit Econ 81:637-654

Björk T, Slinko I (2006) Toward a general theory of good deal bounds. Rev Finance 10:221-260

Carr P, Geman H, Madan D (2001) Pricing and hedging in incomplete markets. J Financial Econ 62:131167

Cerny A, Hodges SD (2000) The theory of good-deal pricing in financial markets, in mathematical financeBachelier Congress 2000. Springer, Berlin

Cerny A (2003) Generalized Sharpe ratios and asset pricing in incomplete markets. Eur Finance Rev 7:191233

Cherny AS (2007) Pricing and hedging European options with discrete-time coherent risk. Finance Stoch 11(4):537-569

Cherny AS (2007) Pricing with coherent risk. Probab Theory Appl 52(3):506-540

Cochrane JH, Saa Requejo J (2000) Beyond arbitrage: good deal asset price bounds in incomplete markets. J Polit Econ 108:1-22

Föllmer H, Schied A (2004) Stochastic finance: an introduction in discrete time. De Gruyter studies in mathematics, vol 27, 2nd edn, Berlin

Grüne L, Semmler W (2008) Asset pricing with loss aversion. J Econ Dyn Control 32:3253-3274

Jaschke S, Küchler U (2001) Coherent risk measures and good deal bounds. Finance Stoch 5:181-200

Kallsen J (2000) Utility-based derivative pricing in incomplete markets, in mathematical finance-Bachelier Congress 2000. Springer, Berlin

Kahneman D, Tversky A (1979) Prospect theory: an analysis of decision under risk. Econometrica 47:263291

King AJ (2002) Duality and martingales: a stochastic programming perspective on contingent claims. Math Program Ser B 91:543-562

Klöppel S, Schweizer M (2007) Dynamic indifference valuation via convex risk measures. Math Finance 17:599-627

Larsen K, Pirvu T, Shreve SE, Tütüncü R (2004) Satisfying convex risk limits by trading. Finance Stoch 9:177-195

Longarela IR (2002) A simple linear programming approach to gain, loss and asset pricing. Topics in theoretical economics, vol 2, Iss. 1, Article 4

Merton RC (1973) Theory of rational option pricing. Bell J Econ Manage Sci 4:141-183

Oberman A, Zariphopoulou T (2003) Pricing early exercise contracts in incomplete markets. Comput Manage Sci 1:75-107

Pınar MÇ, Altay-Salih A, Camcı A (2010) Expected gain-loss pricing and hedging of contingent claims in incomplete markets by linear programming. Eur J Oper Res 201:770-785

Pınar MÇ (2010) Gain-loss pricing under ambiguity of measure. ESAIM Control Optim Calculus Var 16:132-147

Roorda B, Schumacher JM, Engwerda J (2005) Coherent acceptability measures in multiperiod models. Math Finance 15:589-612

Rubinstein M (1976) The valuation of uncertain income streams and the pricing of options. Bell J Econ 7:407-425

Ruszczynski A, Shapiro A (2006) Optimization of convex risk functions. Math Oper Res 31:433-452

Staum J (2004) Fundamental theorems of asset pricing for good deal bounds. Math Finance 14:141-161

$\mathrm{Xu}$ M (2006) Risk measure pricing and hedging in incomplete markets. Ann Finance 2:51-71 